\title{
Impact of connexin 43 coupling on survival and migration of multiple myeloma cells
}

Yangmin Zhang ${ }^{1}$, Ziyan Wang ${ }^{1}$, Liying Zhang ${ }^{1}$, Dongming Zhou ${ }^{1}$, Yu Sun ${ }^{1}$, Panjun Wang', Songguang Ju${ }^{2}$, Ping Chen ${ }^{1}$, Jun $\mathrm{Li}^{1}$, Jinxiang $\mathrm{Fu}^{1}$

${ }^{1}$ Department of Hematology, the Second Affiliated Hospital of Soochow University, Suzhou, China

2Department of Immunology, Medical College of Soochow University, Suzhou, China

Submitted: 5 July 2016

Accepted: 1 November 2016

Arch Med Sci 2017; 13, 6: 1335-1346

DOI: https://doi.org/10.5114/aoms.2017.71065

Copyright @ 2017 Termedia \& Banach

\section{Abstract}

Introduction: Gap junctions (GJs) represent the best known intercellular communication (IC) system and are membrane-spanning channels that facilitate intercellular communication by allowing small signaling molecules to pass from cell to cell. In this study, we constructed an amino terminus of human Cx43 (Cx43NT-GFP), verified the overexpression of Cx43-NT in HUVEC cells and explored the impact of gap junctions (GJs) on multiple myeloma (MM).

Material and methods: The levels of phosphorylated $\mathrm{Cx} 43(\mathrm{~s} 368)$ and the change of MAPK pathway associated molecules (ERK1/2, JNK, p38, NFKB) were also investigated in our cell models. Cx43 mRNA and proteins were detected in both MM cell lines and mesenchymal stem cells (MSCs). Dye transfer assays demonstrated that gap junction intercellular communication (GJIC) occurring via CX43 situated between MM and MSCs or MM and HUVEC ${ }^{\text {CX43NT }}$ is functional.

Results: Our results present evidence for a channel-dependent modulator action of connexin 43 on the migratory activity of MM cells toward MSCs or HUVECCX43-N was higher than those of spontaneous migration $(p<0.05)$ and protection them from apoptosis in the presence of dexamethasone via cytokines secretion. In the meantime, the migration of MM cells involves an augmented response of p38 and JNK signaling pathway of carboxyl tail of the protein.

Conclusions: Our data suggest that GIIC between MM and MSCs is one of the essential factors in tumor cell proliferation and drug sensitivity, and is implicated in MM pathogenesis.

Key words: multiple myeloma, gap junction intercellular communication, migration, cellular signaling.

\section{Introduction}

Multiple myeloma $(M M)$ is a fatal hematological malignancy that develops within the bone marrow microenvironment and is characterized by the uncontrolled clonal proliferation of malignant plasma cells within the bone marrow. It is known that MM-cell tumorigenesis results from a host of known and unknown genetic anomalies, including non-random translocations of genes that normally function as determinants of cell proliferation or cell survival to regions juxtaposed to active immunoglobulin heavy chain enhancer elements, chromosomal aneuploidy, somatic mutations that further affect oncogenic signaling and loss of

\author{
Corresponding author: \\ Dr. Jinxiang Fu \\ Chair of Hematology \\ Department \\ the Second Affiliated \\ Hospital of \\ Soochow University \\ Suzhou 215004 \\ Jiangsu Province \\ China \\ Phone: +86 51267784066 \\ Fax: +86 51267784066 \\ E-mail: fujinxiang@suda.edu.cn
}


heterozygosity of tumor-suppressor genes. However, it is critical to recognize that even in the setting of a genetic disease, the plasma cell tumor microenvironment (TME) contributes significantly to malignant transformation and pathogenesis [1-3]. The evidence continues to demonstrate that dynamic interactions between the MM cells and their TME profoundly influence the behavior of each other. How this microenvironment becomes so supportive of $M M$, and the contribution and interaction of the various components of the microenvironment to enhancing MM growth, are only beginning to be understood [4-6]. This microenvironment is composed of generically denominated stromal cells which include monocytes/macrophages, mesenchymal stem cells, adipocytes, immune cells, osteoclasts, osteoblasts and endothelial cells. Cell-to-cell contact between bone marrow stromal cells and MM cells has been recently proposed as a pivotal regulatory interaction in the growth and survival of malignant plasma cells $[7,8]$.

Gap junctions (GJs) represent the best known intercellular communication (IC) system and are membrane-spanning channels that facilitate intercellular communication by allowing small signaling molecules to pass from cell to cell [9]. Multiple cell types in the bone marrow microenvironment, which express $\mathrm{C} \times 43$, include monocytes, dendritic cells, B and T lymphocytes, as well as MM cells [10-12]. Cx43 is essential for terminal differentiation of $B$ and $T$ cells when tested in Cx43-deficient mice; however, when Cx43 was missing in bone marrow cells alone, $T$ cell development was normal $[10,13]$. More than 20 connexin genes have been found in human and mouse genomes, and disruption of connexin expression often results in developmental defects and lethality [14]. Besides IC, connexins, especially $\mathrm{C} \times 43$, which has a relatively long $\mathrm{C}$-terminal cytoplasmic domain, may serve as adaptor proteins and interact with the cytoskeleton and signaling molecules such as $\beta$-catenin, Src, protein kinase C, and MAPK [15]. Our previous studies [16] also demonstrated that osteoblasts induced from bone marrow mesenchymal stem cells (MSCs) supported survival and proliferation of MM cells. However, the specific role of $\mathrm{Cx} 43$ in the survival and migration of MM cells remains largely unknown. Therefore, in order to investigate the role of GIC between MM and stromal cells, we constructed Cx43-NT over-expressing cells and attempted to determine whether the signaling mediated by the Cx43 C-terminal contributes to $M M$ cell survival and migration and whether bone marrow stromal cell mediated GJ intercellular communication (GIIC) was responsible for the MM clonal expansion but no cytokine production of activated TME.

\section{Material and methods}

\section{Cell lines and primary specimens}

The myeloma cell line RPMI-8226 was obtained from the American Type Culture Collection (ATCC, Manassas, VA, USA) and cultured in RPMI-1640 medium supplemented with $10 \%$ FCS and $1 \%$ glutamine. Interleukin- 6 dependent human myeloma cell lines XG1 were kind gifts from Prof. Zhang XG of Soochow University.

Primary MSCs were obtained from MM patients after informed consent was obtained, accord with the guidelines of the ethics committee of Soochow University Hospital. Four heparinized BM aspirates were collected from MM patients and the bone marrow mononuclear cells (BMNCs) were separated by Ficoll-Hypaque density gradient centrifugation. To purify the MSCs from BMNCs and remove hematopoietic stem cells, CD45+ cells were depleted by negative immunomagnetic cell selection using the Mini MACs device (Miltenyi Biotec, Germany) according to the manufacturer's instructions. Samples obtained before and after depletion of the $\mathrm{CD} 45^{+}$cells were analyzed by flow cytometry (FCM). The resulting CD45-depleted BMNCs were cultured as described previously [16] and the cells were used as Cx43 positive controls.

\section{RNA extraction and PCR assays}

RNA expression of Cx43 of 4 MSCs isolated from MM patients and MM cell lines was carried out using standard RT-PCR techniques as previously described [16]. The primers of the Cx43 were used as follows: Cx43 (BC026329) F:ATGAGCAGTCTGCCTTTCGT and R:TCTGCTTCAAGTGCATGTCC. Cells were washed 2-4 times with PBS prior to RNA isolation. Briefly, total RNA was extracted using the RNeasy mini kit (Qiagen, Germany) and contaminating genomic DNA was removed by treatment with RNase-free DNase I (Qiagen, Germany). The PCR was optimized by $1.5 \mathrm{mmol} / / \mathrm{MgCl}_{2}$ and $0.5 \mathrm{U}$ of Platinum Taq DNA polymerase in a $20-\mu \mathrm{l}$ reaction. Amplification conditions included $35 \mathrm{cy}-$ cles at $94^{\circ} \mathrm{C}$ for $50 \mathrm{~s}, 62^{\circ} \mathrm{C}$ for $20 \mathrm{~s}, 72^{\circ} \mathrm{C}$ for $20 \mathrm{~s}$, and a final extension of $10 \mathrm{~min}$ at $72^{\circ} \mathrm{C}$ in a thermocycler (Eppendorf, Hamburg, Germany); $\beta$-actin served as an internal control.

\section{Construction of Cx43-NT lentiviral vectors}

Molecular cloning was done by Shanghai Novobio Inc. China. DNA construct coding for amino acids 1-231 of the amino terminus of human $C \times 43$ (Cx43NT-GFP) construction and verifying the overexpression of Cx43-NT in HUVEC cells were carried out using standard techniques as previously described [17, 18]. Briefly, for expression in HUVEC 
cells, a full length cDNA of amplified human Cx43 ${ }^{\text {WT }}$ cDNA was cloned into a pCDNA3.1 vector (Invitrogen, USA) using a designed oligonucleotide primer pair, 5'-ATGAAGCTTATGGATTGGGGGACGCTG-3' and 5'-ATGGCGGCCGCTTAGCTTGGGAAA CCTGTGAT-3', to generate 5' HindIII (aagctt) and 3' Notl (gcggccgc) restriction sites for inframe ligation. The oligonucleotide primer pair was designed to preferentially amplify the Cx43-NT from the genomic DNA for the HUVEC cell line, which was: CX-NT-R; ACTGCTGGAGGGAAGGTG; CX-NT-F; TGAGTGTTACAACGAAAG; pL/to/v5/GFP/IRES/MCSCX43-NT-R was generated with connection of the Cx43-NT-GFP sequence and lentiviral vectors through double digestion, which were transfected into 293T cells with secondary carriers. Subsequently the collected virus supernatant was infected with HUVEC cells, transfection efficiency of which was observed by fluorescence microscopy and QPCR. All constructs were confirmed by sequencing.

\section{Cell culture, selection and characterization of Cx43-NT overexpressing cells}

The HUVEC cell line (ATCC, Manassas, VA, USA) was maintained in Dulbecco's modified minimal essential medium (DMEM, Life Technologies, Inc., Burlington, ON, USA) supplemented with $10 \%$ (v/v) fetal bovine serum (Life Technologies, Inc.), at $37^{\circ} \mathrm{C}$, in a humidified atmosphere containing $95 \%$ air and $5 \% \mathrm{CO}_{2}$. These cells were used throughout this study. For transfections, the cells were split the day prior to transfection to reach $70-80 \%$ confluence. Stable HUVEC cell transfectants expressing Cx43-NT were generated by transfection, respectively, with a Cx43-NT cDNA encoding plasmid using the Lipofectamine kit (Invitrogen Corporation, California, USA) and subsequent selection for G418 resistance according to the manufacturer's instructions. Transfectants were tested by QPCR for Cx43-NT expression.

\section{GIC between the HUVEC and myeloma cells}

The myeloma cell line RPMI8266 cells were simultaneously labeled with $10 \mu \mathrm{M}$ calcein-AM (Invitrogen, USA) and $10 \mu \mathrm{M}$ 1,1'-dioctadecyl3,3,3',3-tetramethylindo-carbocyanine perchlorate (Dil, Invitrogen, USA) for $25 \mathrm{~min}$ at $37^{\circ} \mathrm{C}$ in a $5 \%$ $\mathrm{CO}_{2}$ fully humidified atmosphere. Cells were washed twice in PBS prior to being dropped onto receiving cells (MSCs and HUVEC ${ }^{\text {CX43-NT }}$ were $>90-$ $95 \%$ confluent). A total of $2 \times 10^{4}$ resulting cells were seeded to the precultured MSCs and HUVEC ${ }^{\text {CX43-NT }}$ cells and the culture was incubated for $75 \mathrm{~min}$ at $37^{\circ} \mathrm{C}$. To block the GIIC, heptanol was dissolved in ethanol as described previously [19, 20], so that the final concentration in the cell cul- ture medium equaled $0.15 \%$ ethanol with 50 and $100 \mathrm{mM}$ heptanol.

Prior to conducting FACS studies, the cultures were examined microscopically to confirm transfer of dye from myeloma cells to MSCS or HUVEC Cx43-NT. For FACS analysis studies, cells were trypsinized to release MSCS and HUVEC ${ }^{\text {Cx43-NT, }}$ fixed in 4\% paraformaldehyde, and resuspended in FACS buffer, and then FACS analyzed on a Facstar Plus Flow Cytometer (Becton Dickinson, USA).

\section{Co-culture of HUVEC with myeloma cell}

To test the effects of $\mathrm{Cx} 43$ coupling on survival of MM cells, RPMI8266 and XG1 cells were plated to 6 -well plates at a concentration of $5 \times 10^{5} /$ well alone or with MSCS and HUVEC Cx43-NT in medium with or without $2 \times 10^{-7} \mathrm{M} / \mathrm{I}$ dexamethasone. The cultures were continued for $48 \mathrm{~h}$ and cell numbers, viability (trypan blue assay) and annexin V/PI binding were determined by flow cytometry. In the meantime, co-culture of RPMI8266 and XG1 MM cells with MSCs and HUVEC ${ }^{\mathrm{C} \times 3-\mathrm{NT}}$ in medium containing $2 \times 10^{-7} \mathrm{M}$ dexamethasone and $50 \mathrm{mM} / \mathrm{ml}$ heptanol was also done in the same conditions.

\section{Transwell migration assay}

We performed a transwell migration assay (Costar) using the MM cell line RPMI 8226 in the presence of MSCS or HUVEC ${ }^{\text {Cx43-NT }}$ cells, which were cultured in the lower chambers. Seven groups were included in the assay: (1) control, upper chamber: MM cells, lower chamber: DMEM-LG supplemented with $0.5 \%$ FBS; (2) MSCs; upper chamber: MM cells, lower chamber: MSCs; (3) MSCs + heptanol; upper chamber: MM cells, lower chamber: MSCS + heptanol; (4) HUVEC ${ }^{\text {Cx43-NT; }}$ upper chamber: MM cells, lower chamber: HUVEC ${ }^{\mathrm{C} \times 43-\mathrm{NT}}$; (5) HUVE ${ }^{\mathrm{CX} 43-\mathrm{NT}}$ + heptanol; upper chamber: MM cells, lower chamber: HUVEC ${ }^{\mathrm{C} \times 43-\mathrm{NT}}$ + heptanol. In brief, MM cells were suspended in $0.5 \%$ FBS medium, and $5 \times 10^{5}$ cells were placed in the upper chambers of the transwell plates with or without MSCS or HUVEC CX43-NT cells in the lower chambers. The concentration of heptanol used in these experiments was $50 \mathrm{mM} / \mathrm{l}$. After $4 \mathrm{~h}$ at $37^{\circ} \mathrm{C}$, cells that migrated to the lower chambers were counted. Triplicate experiments were performed with each group, and the means and standard deviations were calculated.

\section{Cytokine production}

The supernatants from RPMI8266 cells alone or with MSCS and HUVEC CX43-NT in medium with or without heptanol $(50 \mathrm{mM} / \mathrm{l})$ were collected. The IL-6, IL-10, transformating growth factor $\beta$ (TGF- $\beta$ ), and SDF- $1 \alpha$ concentrations were measured by Cytometric Bead Array (CBA) assay, using kits obtained from BD Biosciences Pharmingen 
(USA) according to the manufacturer's recommendations.

\section{Western blotting}

RPMI8266 cells alone or with MSCs and HUVEC ${ }^{\text {C43-NT }}$ in medium with or without heptanol $(50 \mathrm{mM} / \mathrm{l})$ from direct co-culture systems were collected according to the methods mentioned above. Total cell lysates for Western blots were prepared by lysing cell pellets in radioimmunoprecipitation assay (RIPA) buffer. Bicinchoninic acid was used to determine protein concentrations. The lysates were separated by sodium dodecyl sulfate-polyacrylamide gel electrophoresis (SDS PAGE; Bio-Rad, USA), and transferred to polyvinylidene difluoride membranes, which were subsequently blocked in Tris-buffered saline containing 5\% non-fat milk and $0.1 \%$ Tween for $1 \mathrm{~h}$. The membranes were then incubated with polyclonal rabbit anti-p-Cx43 hSer368, ERK1/2, JNK, p38, NFKB (the antibodies were bought from Cell Signaling Technology) and anti-glyceraldehyde 3-phosphate dehydrogenase (GAPDH) (Sigma) at $4^{\circ} \mathrm{C}$ overnight. Antigen-antibody complexes were detected using secondary antibodies conjugated to horseradish peroxidase (Invitrogen) and visualized by enhanced chemiluminescence (GE Healthcare, UK).

\section{Statistical analysis}

Statistical analyses of data were performed using the Statistical Package for the Social Sciences (SPSS) software version 17.0 (SPSS Inc., Chicago, IL, USA). Differences were evaluated by Student's $t$ test and nonparametric Mann-Whitney test (significance cutoff: $p<0.05$ ).

\section{Results}

Cx43 expressed differently among the $M M$ cell lines and primary MM cells

The expression of Cx43 on MM cell lines RPMI 8226 and $X G 1$ and the MSCs isolated from MM patients $(n=4)$ was determined using PCR assays. The results showed that both MM cells and MSCs expressed $\mathrm{C} \times 43$, although the expression of $\mathrm{C} \times 43$ in those cells was at different levels (Figure $1 \mathrm{~A}$ ). Western blot analysis revealed that RPMI 8226 and XG1 cells expressed $\mathrm{CX} 43$ at moderate levels. All four primary MSCs from MM patients expressed Cx43 (Figure 1 B). Generally, Cx43 expression in MSCs was stronger than that in MM cells $(p<0.05)$.

\section{GJIC between MM cells and HUVEC are functional}

Transfectants were confirmed by QPCR for Cx43-NT expression (Figure 2). To clarify the role of Cx43 on MM cells, we employed Cx43-NT truncated Cx43 to overexpress on HUVEC cells and MSCs isolated from MM patients as positive controls.

To determine whether MM cells couple with HUVECS, MM cells, MSCs and HUVEC CX43-NT cells were cocultured and examined microscopically to confirm transfer of dye from MM cells to MSCs or HUVEC ${ }^{\text {Cx43-NT }}$ cells, although there was much less dye transferred to HUVEC ${ }^{\mathrm{C} \times 43-\mathrm{NT}}$ as comparison to MSCs. Visual inspection confirmed the viability of both the donor and receptor cells and demonstrated that the dye transfer was specific. FACS analysis was used to show the transfer of calcein AM to MSCs and HUVEC Cx43-NT (Figure 3), demonstrating that MM-HUVEC GIIC occurs.

Because our data showed that MM can couple with MSCs and HUVEC CX43-NT cells, we wanted to confirm that the GJIC was specific. This was accomplished by using the GJ blocking agent. Heptanol was titrated into HUVEC cultures and then the cultures were analyzed by FACS analysis as above. Our results demonstrated that inhibition of dye transfer from MM to MSCS or HUVEC ${ }^{\mathrm{C} \times 43-\mathrm{NT}}$ occurred in a dose-dependent manner.

\section{Coupling with MSCS/HUVEC ${ }^{\mathrm{C} \times 43-\mathrm{NT}}$ protected MM cells from apoptosis in presence of dexamethasone}

RPMI 8266 and XG1 MM cells were incubated alone or cultured with MSCS and HUVEC Cx43-NT for $48 \mathrm{~h}$ in medium with or without dexamethasone. The total number of myeloma cells recovered from cultures of myeloma cells alone was significantly lower than that from co-cultures, and their viability was also significantly lower $(p<0.01)$, while their apoptotic rate was significantly higher than that of myeloma cells cultured with MSCs and HUVEC ${ }^{C 43-N T}(p<0.01)$ (Figure 4). Both MSCs and
A

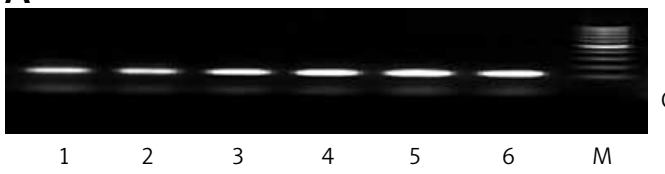

B

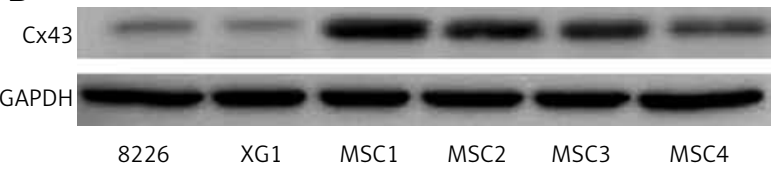

Line 1-2 MM cells, Line 3-6 MSCs

Figure 1. Cx43 expression on MM cells and MSCs. The RT-PCT assays showed that either MM cell lines or primary MSCs isolated from MM patients expressed Cx43 (A). Western blot assay showed that MSCs expressed higher levels of Cx43 than those of MM cells (B) 

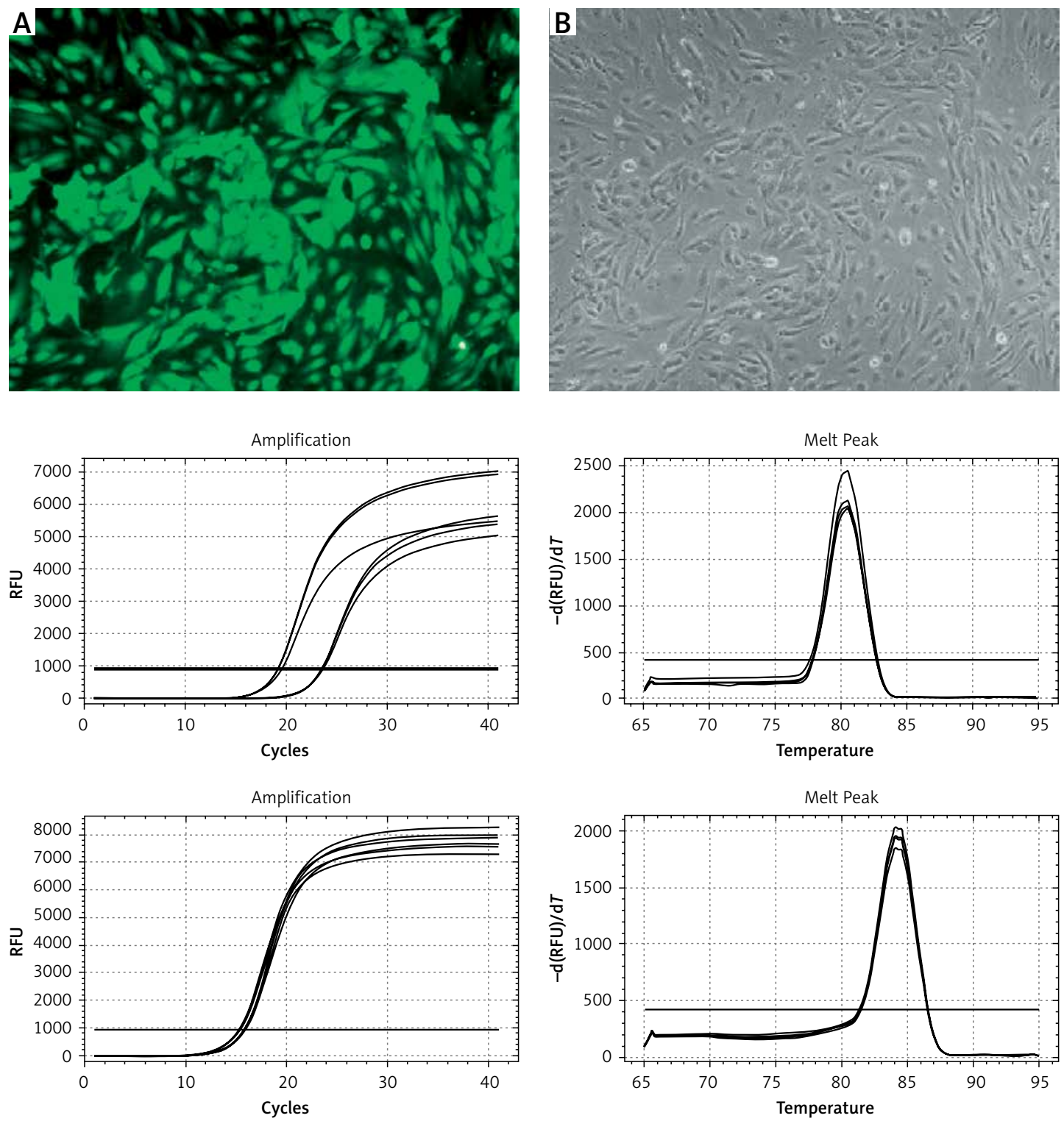

Figure 2. Expression of Cx43-NT in HUVEC cell lines. Microscopic photographs show the expression of Cx43-NTGEP in HUVEC cells (A) and control (B). Total DNA was prepared from the cells and transfectants were confirmed by real-time PCR

HUVEC Cx43-NT could partially rescue the MM cells from apoptosis in the co-culture conditions. It seemed that MSCs were more effective to protect $M M$ cells from apoptosis in medium containing dexamethasone and that XG1 cells was more sensitive to dexamethasone-induced cell death. Our results demonstrated that the GJs have an impact on the survival of MM cells and more MM cells were apoptotic in the medium containing the GJ inhibitor heptanol.

\section{GJ blockage by heptanol inhibited} migration of MM cells induced by MSCS

In transwell migration assays, the migration rate of MM cell lines (RPMI 8226, XG-1) toward MSCS HUVEC ${ }^{\mathrm{C} \times 43-\mathrm{N}}$ was higher than that of spon- taneous migration $(p<0.05)$, which indicated that both MSCS and HUVEC ${ }^{\mathrm{C} \times 43-\mathrm{NT}}$ could induce the migration of MM cells. The migration of MM cells toward MSCs was the highest among the 5 groups $(p<0.05)$. The process was partially inhibited by the GJ inhibitor heptanol (Figure 5). These results show that heptanol can inhibit the migration of MM cells induced by MSCs or endothelial cells (HUVEC) and indicate that GJs may play important roles in the process of chemotaxis induced by MSCS and HUVEC cells.

\section{Cx43 coupling changes the cytokine prolife of MSCs}

CBA assays revealed high levels of IL- 6 in supernatant of MSCS cultured alone, but the others 

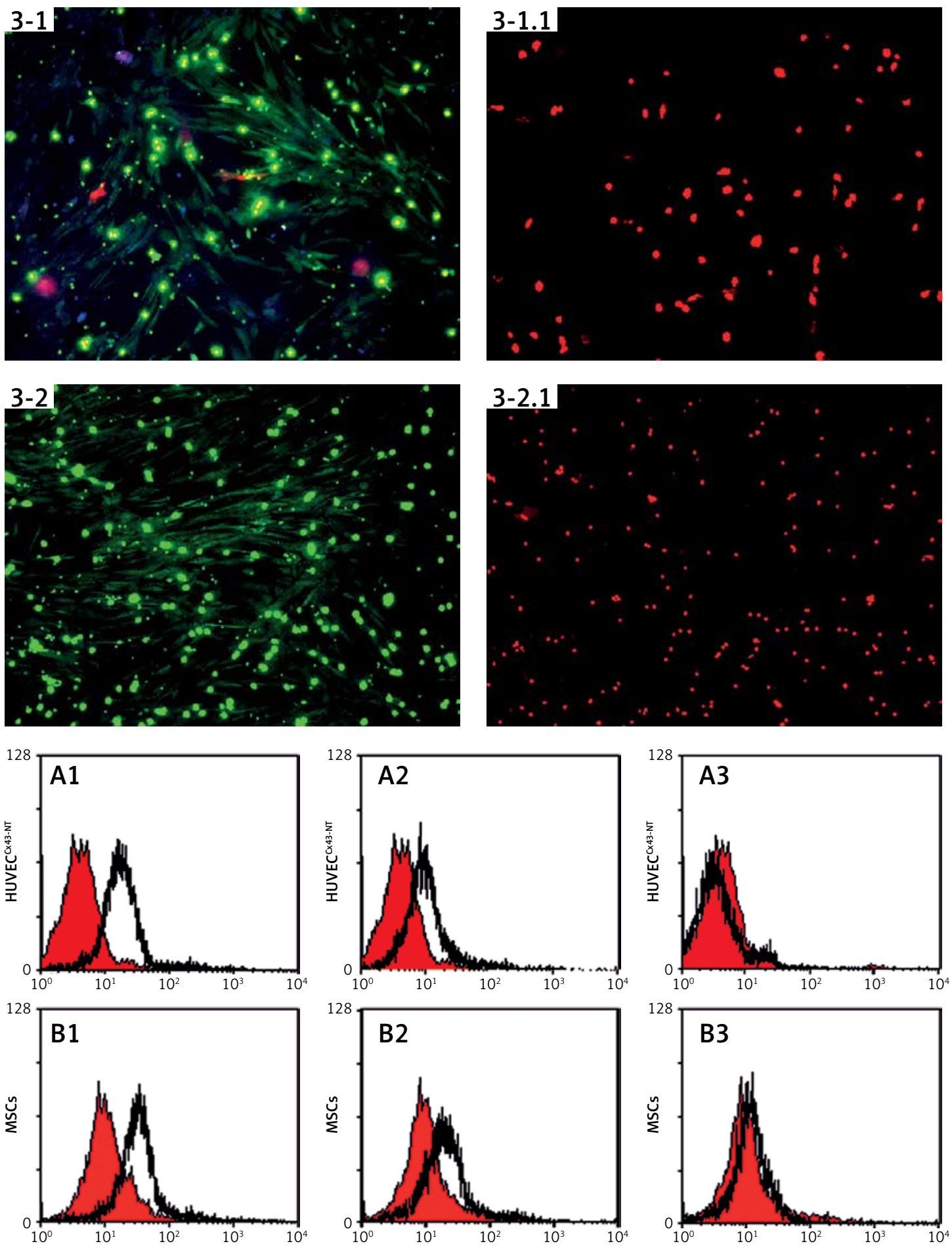

Figure 3. Gap junctions among the MM, MSCs and HUVEC Cx43-NT. Microscopic photographs from the double labeling of MM cells with calcein-AM (green) and Dil (red) show that dye transfer occurs from myeloma cells to MSCs and $\operatorname{HUVEC}^{\mathrm{C} \times 43-\mathrm{NT}}(3-1 / 3-2)$ and that dye does not permeate from the cells stained with Dil (3-1.1/3-2.1), confirming that GIIC is functional between the two cells, and that the dye transfer was specific. FCM histograms (A, B) of RPMI 8266 after dual-labeled MM were administered onto MSCs in a parachute assay, with GJs allowed to form. FCM data show the percentage of MSCs into which calcein-AM was transferred from MM cells and was inhibited in the presence of heptanol $(50 \mathrm{mM} / \mathrm{l})$ 

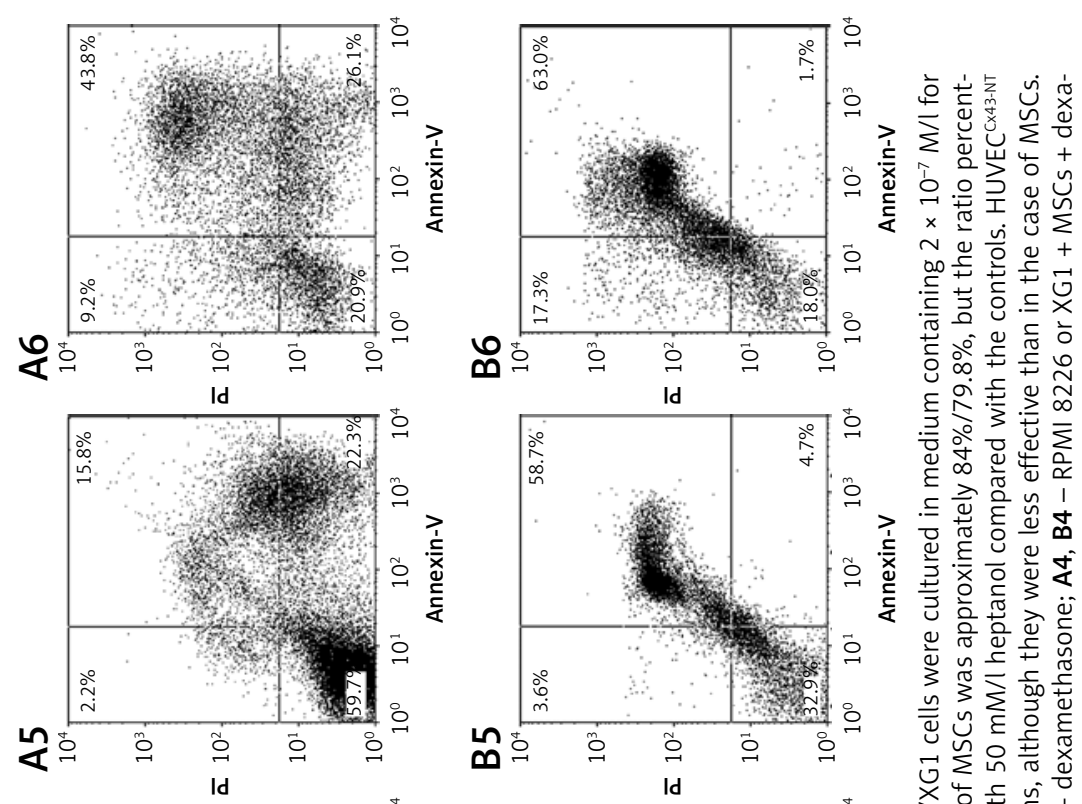

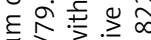
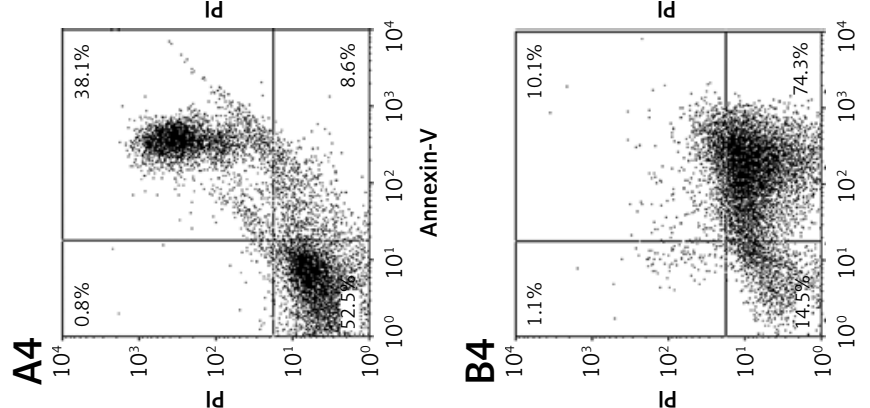

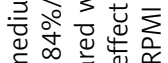

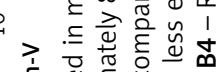

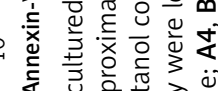
ष

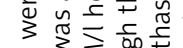

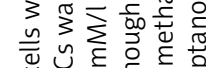

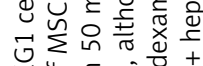
$\times$ 约
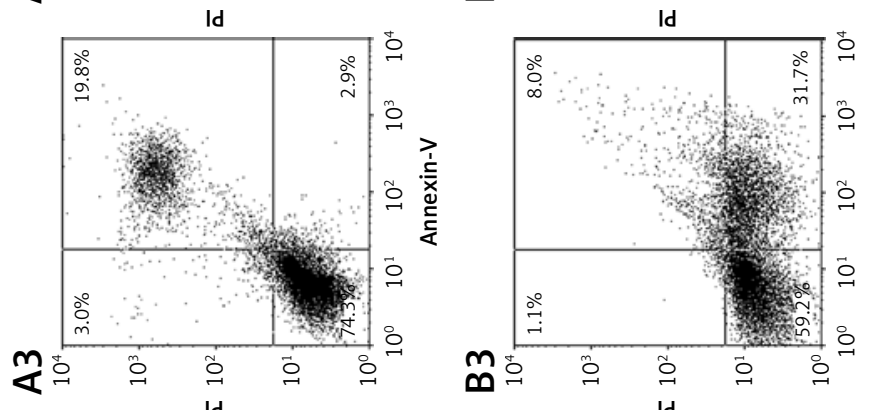

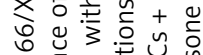

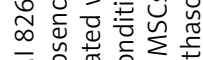
$>\sum_{0} \pi 00$

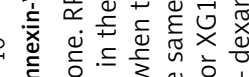
o

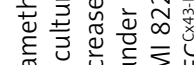
芉产.

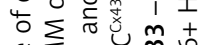

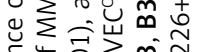

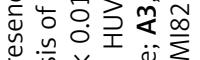

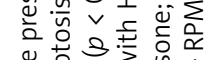

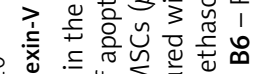

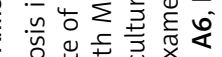

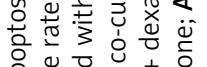

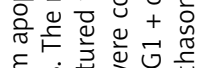

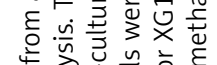

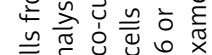
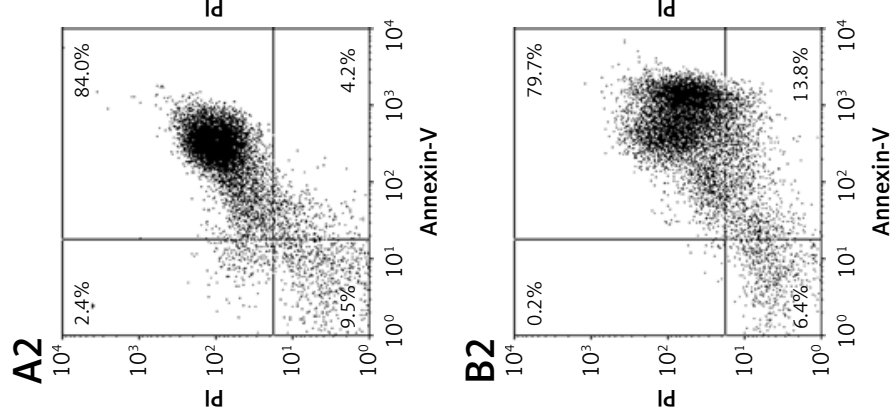

ฮั $\sum_{\sum} \sum_{u}^{\infty} \sum_{i}^{\infty} \sum_{0}^{\infty} \infty+$

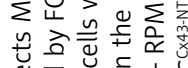

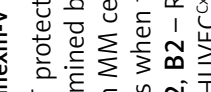

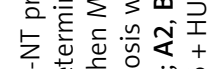

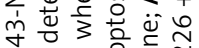

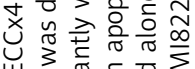

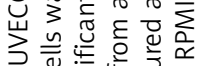
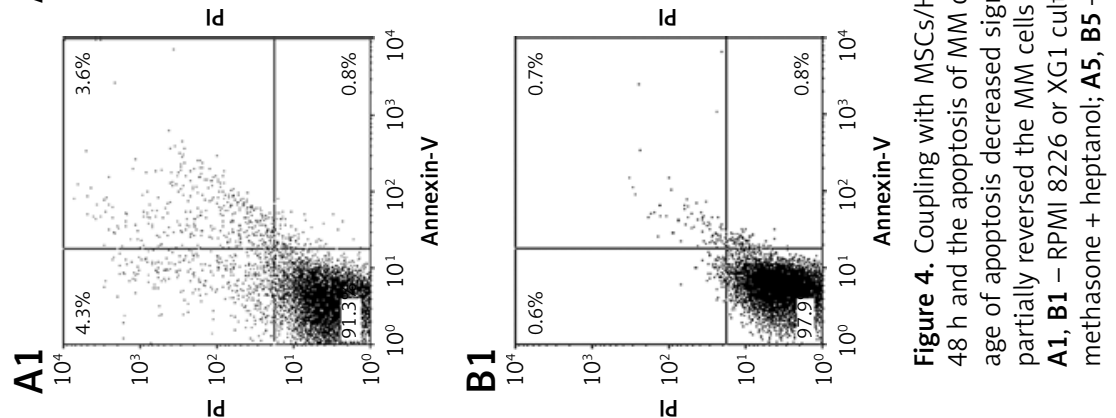


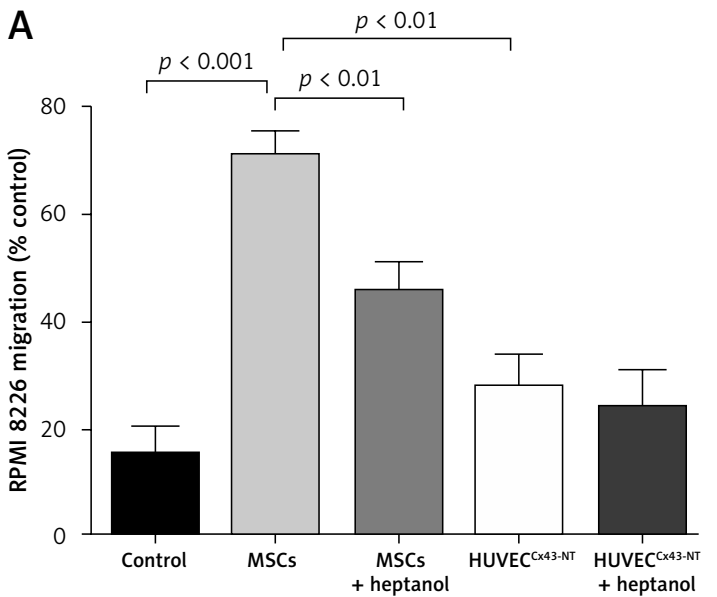

Figure 5. Effects of GJ inhibitor on migration of RPMI 8226

were at low levels, and there was no IL-10 detected in the supernatant. The cytokines detected in the supernatant of RPMI 8266 cultured alone was also at a low level. The concentration of cytokines detected in supernatant of HUVEC CX43-NT was low in comparison with MSCs, but higher than that of RPMI 8226. The level of IL-6, IL-10 and SDF- $1 \alpha$ increased significantly after MSC and MM cells were co-cultured for $24 \mathrm{~h}(p<0.01)$, and especially the levels of IL-6 and IL-10 were higher in comparison to those cells cultured alone $(p<0.001)$. There was no change of the level of TGF- $\beta$ before and after

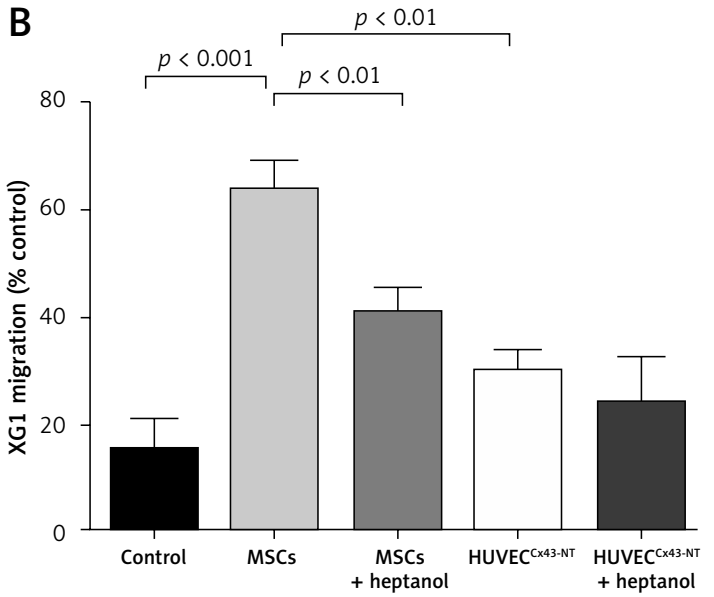

6 (A)/XG1 (B) cells induced by MSCS or HUVEC ${ }^{\mathrm{C} \times 43-\mathrm{NT}}$

co-culture. After blockage of the GIIC with heptanol, the levels of IL- 6 and SDF- $1 \alpha$ returned to normal, but the level of IL-10 was higher than in those cells cultured alone $(p<0.01)$. The same results were also obtained from RPMI 8226 cells co-cultured with HUVEC CX43-NT. The levels of cytokines were lower than those of MSCs $(p<0.05)$ (Figure 6).

\section{GJ between MM cells and stromal cells involved in JNK and NF- $K B$ signaling}

In this study, in order to explore further the molecular mechanisms of Cx43 signaling in the

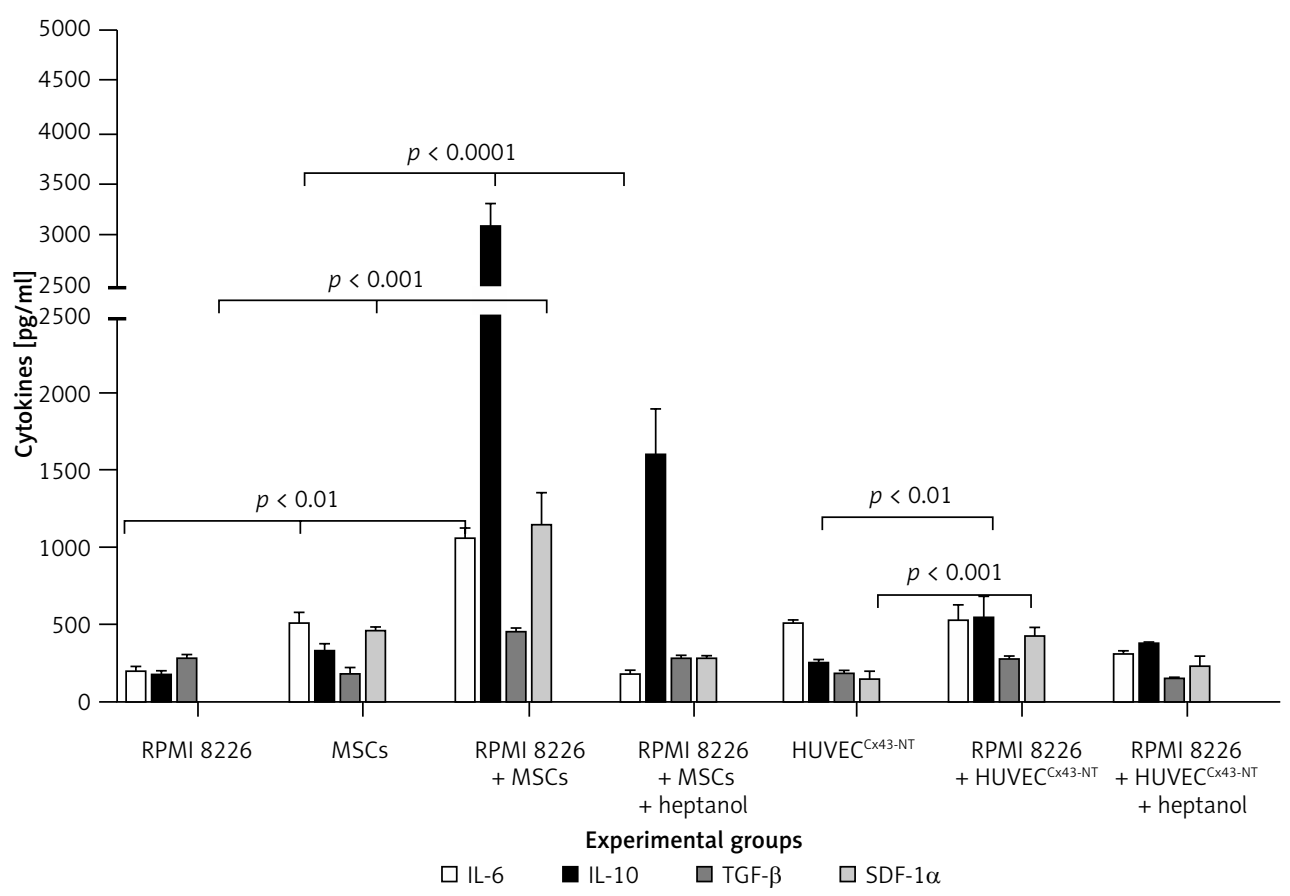

Figure 6. The change of cytokines in supernatant of RPMI 8226 MM cells co-cultivated with different cells with or without GJ inhibitor heptanol. We demonstrated that the cytokine profiles of MSCs changed when they were incubated with MM cells and MM promoted the MSCs to secrete the cytokines, which was in favor of the homing or growth of MM cells. The levels of IL-6, IL-10 and SDF-1 $\alpha$ increased significantly after MSCs and MM cells were co-cultured for $24 \mathrm{~h}(p<0.01)$ and decreased in the presence of the GJ inhibitor heptanol. We also found that the cytokines secreted by HUVEC CX43-NT changed and the levels of IL-10 and SDF-1 $\alpha$ increased in co-culture and decreased in the presence of heptanol 
MM, we investigated the changes of signaling pathways within our cell model during the Cx43 coupling in the presence and absence of the $\mathrm{C} \times 43$ specific inhibitor heptanol. As shown in Figure 7, the protein levels of phosphorylated $\mathrm{C} \times 43$ were increased in MSCs after co-culture and $\mathrm{pC} \times 43$ decreased significantly in the presence of the G) inhibitor. However, the pCx43 expression of HUVEC ${ }^{\mathrm{C} \times 43-\mathrm{NT}}$ showed no marked changes whether in co-culture with MM cells or in the presence of heptanol. To understand whether the MAPK intracellular signaling molecules might be involved in the C-terminus of Cx43 in mediating the transduction of cell survival signals and thereby exerting

A

8226 MSCS HUVEC ${ }^{C \times 33-N T}$

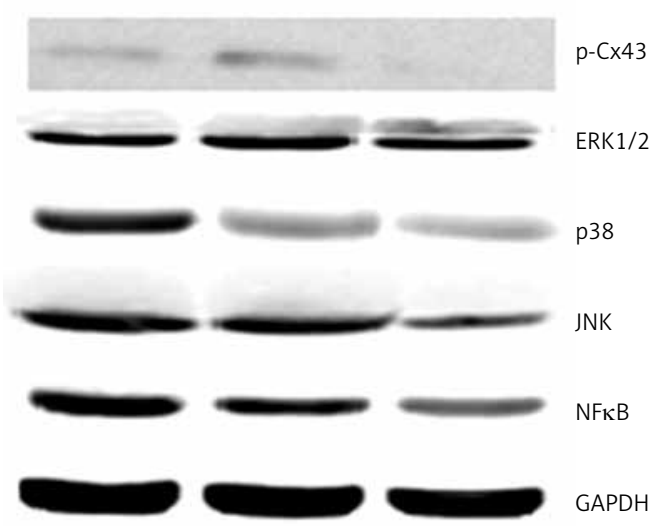

anti-proliferative effect on MM cells, we investigated the change of MAPK pathways by Western blot assay in our cell models. We found that the expression of p38, JNK and NFKB increased, and especially the expression of p38 and JNK was significantly increased in co-culture, while it had no effect on the expression of ERK $1 / 2$. Our study showed that $\mathrm{C} \times 43$ coupling promotes MM cell survival via activation of p38, JNK and its downstream signal pathway.

\section{Discussion}

GIC has been implicated in the regulation of homeostasis and a diverse array of cellular func-

B
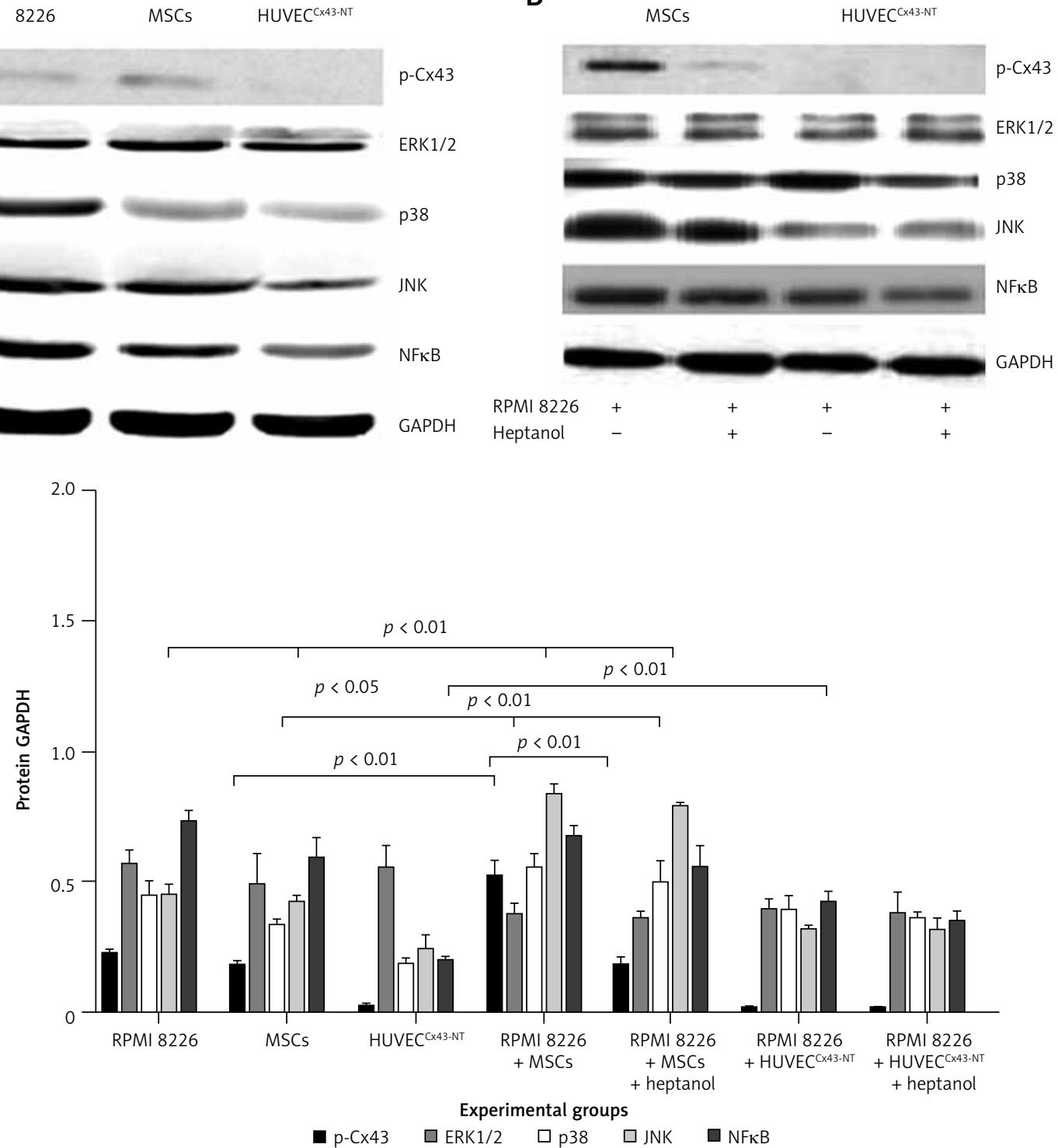

Figure 7. The change of signaling molecules in medium containing GJ inhibitor in co-culture. We demonstrated that the MM coupling with MSCs enhanced the levels of phosphorylated Cx43 at the C-terminus and decreased in the medium containing GJ inhibitor $(p<0.01$ ). The expression of $\mathrm{p} 38$, JNK and NFKB also increased; especially the expression of $\mathrm{p} 38$ and JNK was significantly increased in co-culture. We conjectured that the MAPK signaling pathway might be involved in crosstalk between them. Results are reported as relative quantitation of target molecule expression, normalized to an endogenous control (GAPDH) and relative to a calibrator 
tions related to cell specialization, growth and differentiation [21]. Altered expression of connexins, such as reduced expression or aberrant localization, has been reported in many tumor tissues [2225]. Such alterations prevent cell-to-cell adhesion, which is indispensible for the formation of gap junctions. In consequence, a clone of cells selectively acquires the ability for fast and unrestricted growth along with disruption of tissue integrity. Recent evidence further suggests that connexins may even facilitate late stage disease progression and metastasis [26]. Although connexins seem to be linked to tumorigenesis in the breast $[21,27$, 28], the current understanding of how these molecules are linked to cancer is relatively limited, as few studies have systematically examined the role of connexins in both early and late stages of disease progression in a unified model.

The results of many studies indicate that the microenvironment created by MSCs plays an important role in supporting myeloma cells. Although it has been shown that myeloma stromal cells contribute to mature myeloma cell growth and chemoresistance, little is known about the GJ interplay between MSCs [29, 30]. We have previously demonstrated that $\mathrm{C} \times 43$ was expressed heterogeneously and aberrantly in MM cells. Interestingly, Cx43 expression in MSCs isolated from patients with MM was greater than that observed in both MM cells and normal MSCs [31]. To determine biochemically the function of the CX43 protein within the cells and elucidate the role of CX43 in gap junction formation, this study cloned and expressed the $\mathrm{N}$-terminal part of $\mathrm{CX} 43$ in HUVEC cells. The studies reported herein showed that both MM cell lines and MSCs isolated from MM patients express $\mathrm{C} \times 43$ at mRNA and protein levels, and GJ formed between those cells was functional. MM cells also coupled with HUVEC ${ }^{\text {CX43-NT }}$ cells and formed a functional GJ between them. We confirmed the existence of GIIC among MM cells, MSCS and HUVEC ${ }^{\text {CX43-NT }}$ cells by a dye transfer assay, which occurred in a dose-dependent manner and was blocked with the GJ-specific inhibitor heptanol. Cx43 is required for $M M$ cell survival and partially reversed the apoptosis induced by dexamethasone, as evidenced by the ability of the GJ inhibitor heptanol to enhance the $M M$ cell apoptosis in the same conditions. We conjectured that the GIIC between MM cells and MSCs may be one of the ways involved in the crosstalk in the bone marrow niche and may have a significant impact on MM biology.

Multiple myeloma cells express various adhesion molecules such as LFA-1 and VLA-4 [32]. These molecules bind to their cognate receptor/ adhesion molecule on the surface of the MSCs and thus play an important role in the MM cells' and marrow stromal cells' interactions in vivo and in vitro [33]. Adhesion of the MM cells to MSCs activates many pathways, resulting in promotion of cell migration regulating proteins, up-regulation of numerous cytokines secreted by both $M M$ cells and MSCs and anti-apoptotic proteins in the MM cells [34]. Our results indicated that GJ is one important way to activate the MSCs to secrete cytokines and MM cells to migration. CBA assays revealed that there were high levels of IL-10 in supernatant of MSCs cultured alone, but the others were at low levels, and the levels of IL- 6 were moderately elevated in the supernatant. The cytokines detected in the supernatant of PMI 8266 cultured alone were at a low level. The levels of IL- 6 , IL-10 and SDF-1 $\alpha$ increased significantly after MSC cells were co-cultured with MM cells for $24 \mathrm{~h}$ ( $p<$ 0.001 ); in particular, the level of IL-10 was higher than in those cells cultured alone $(p<0.0001)$. After blockage of the GJ with heptanol, the levels of IL- 6 and SDF- $1 \alpha$ returned to normal, but the level of IL-10 was higher than in those cells cultured alone $(p<0.01)$. There was no change of the level of cytokines before and after co-culture in the supernatant of HUVEC ${ }^{\text {CX43-NT }}$ cells with or without heptanol except the levels of SDF-1 $\alpha$. It has been confirmed that SDF- $1 \alpha$ secreted by MSCs is one of the main chemokines that can induce MM cell homing and localization selectively in bone marrow. We used heptanol, a type of GJ inhibitor that can inhibit GJs composed of many types of adherens proteins including $\mathrm{C} \times 43$, to study the role of Cx43 in the migration of MM cells in a transwell migration assay, in which MSCs could induce MM cell migration and the process could be inhibited by heptanol. Meanwhile, we found that the level of SDF-1 $\alpha$ was higher in the supernatant of MSCS co-cultured with MM cells compared with that of MSCs cultured alone. This was also in agreement with observations showing that connexins expression may be present in later stages of carcinogenesis, favoring the migration capacity of invasive cells [35]. The Cx43 without the C-terminal lost the function of enhancing the migration of $M M$ cells and the GJ inhibitor had no effects on the migration of MM cells. Although there seemed to be more cells counted in the lower chamber, the difference was not statistically significant.

Though the transmembrane part and the extracellular loops are highly conserved among the different connexin isoforms, the carboxyl terminus is the most variable in length and amino acid sequence. The carboxyl terminus of several connexins contains various phosphorylation sites which play an important role in regulating GJ channel conductivity and function. Recently, a channel-independent role for connexins in intracellular signaling was suggested. Plotkin et al. 
[36-38] demonstrated a novel function of the C-terminus of $\mathrm{Cx} 43$ in mediating the transduction of cell survival signals induced by the anti-osteoporosis drugs bisphosphonates in osteoblasts and osteocytes. Their studies showed that bisphosphonates open $\mathrm{Cx} 43$ hemichannels and induce the interaction of $\mathrm{C} \times 43$ with the kinase Src, followed by activation of the ERK pathway and inhibition of osteoblast and osteocyte apoptosis. This evidence raises the possibility that a similar scaffolding function of $\mathrm{C} \times 43$ could be required for the survival effect of MM cells. Our results demonstrated that the protein levels of phosphorylated Cx43 were increased in MSCs after co-culture with MM cells and $p C \times 43$ decreased significantly in the presence of a GJ inhibitor (Figure 7). However, the $p C \times 43$ expression of HUVEC ${ }^{\text {Cx43-NT }}$ showed no marked changes whether in co-culture with MM cells or in the presence of heptanol. To understand the MAPK intracellular signaling molecules which might be involved in the C-terminus of $\mathrm{Cx} 43$ in mediating the transduction of cell survival signals so as to exert an anti-apoptotic effect on MM cells, we investigated the change of MAPK pathway associated molecules by western blot assay in our cell models. We found that the expression of p38, JNK and NFKB increased, and especially the expression of p38 and JNK was significantly increased in co-culture, while it had no effect on the expression of ERK1/2, and the up-regulated p38 and JNK expression was not inhibited in the presence of the GJ inhibitor. We conjectured that the adhesion of MM cells to MSCs may be involved apart from GJ in the crosstalk between them. Behrens et al. [39] demonstrated that expression of Cx43 augments migration via modulation of p38 MAP kinase activity. The carboxyl tail of Cx43 plays an essential role in this signaling pathway, which is independent of gap junction function. Our study was consistent with theirs and confirmed that Cx43 coupling promoted MM cell survival via activation of $\mathrm{p38}$, JNK and its downstream signal pathway. Recently, Belting et al. [40, 41] demonstrated the potential role of nanotubes, exosomes, apoptotic bodies, and nucleic acid-binding peptides in the intercellular transfer of genetic information and indicated that various facets of cell biology research will revolutionize the way we perceive how cells communicate as well as the way we treat various diseases. In that respect, a better understanding of the interaction of the MM cells with bone marrow cells would provide insight into a wide array of pathophysiologies and whether this opens avenues for therapeutic intervention.

\section{Conflict of interest}

The authors declare no conflict of interest.

\section{References}

1. Klein U, Dalla-Favera R. Germinal centres: role in B-cell physiology and malignancy. Nat Rev Immunol 2008; 8: 22-33.

2. Mitsiades CS, McMillin DW, Klippel S, et al. The role of the bone marrow microenvironment in the pathophysiology of myeloma and its significance in the development of more effective therapies. Hematol Oncol Clin N Am 2007; 21: 1007-34 vii-viii.

3. Azab AK, Hu J, Quang P, et al. Hypoxia promotes dissemination of multiple myeloma through acquisition of endothelial to mesenchymal transition-like features. Blood 2012; 119: 5782-94.

4. Zhan F, Hardin J, Kordsmeier B, Bumm K, Zheng M, Tian E. Global gene expression profiling of multiple myeloma, monoclonal gammopathy of undetermined significance, and normal bone marrow plasma cells. Blood 2002; 99: 1745-57.

5. Keats JJ, Reiman T, Belch AR, Pilarski LM. Ten years and counting: so what do we know about $t(4 ; 14)$ (p16;q32) multiple myeloma. Leuk Lymphoma 2006; 47: 2289-300.

6. Fowler JA, Edwards CM, Croucher PI. Tumor-host cell interactions in the bone disease of myeloma. Bone 2011; 48: 121-8.

7. Fowler JA, Mundy GR, Lwin ST, Lynch CC, Edwards CM. A murine model of myeloma that allows genetic manipulation of the host microenvironment. Dis Model Mech 2009; 2: 604-11.

8. Corre J, Mahtouk K, Attal M, et al. Bone marrow mesenchymal stem cells are abnormal in multiple myeloma. Leukemia 2007; 21: 1079-88.

9. Civitelli R. Cell-cell communication in the osteoblast/ osteocyte lineage. Arch Biochem Biophys 2008; 473: 188-92.

10. Montecino-Rodriguez, Leathers EH, Dorshkind K. Expression of connexin $43(C \times 43)$ is critical for normal hematopoiesis. Blood 2000; 96: 917-24.

11. Presley CA, Lee AW, Kastl B, et al. Bone marrow connexin-43 expression is critical for hematopoietic regeneration after chemotherapy. Cell Commun Adhes 2005; 12: 307-17.

12. Wong RCB, Pera MF, Pébay A. Role of gap junctions in embryonic and somatic stem cells. Stem Cell Rev 2008; 4: 283-92.

13. Nguyen TD, Taffet SM. A model system to study connexin 43 in the immune system. Mol Immunol 2009; 46: 2938-46.

14. Laird DW. Life cycle of connexins in health and disease. Biochem J 2006; 394: 527-43.

15. Herve JC, Bourmeyster N, Sarrouilhe D, Duffy HS. Gap junctional complexes: from partners to functions. Prog Biophys Mol Biol 2007; 94: 29-65.

16. Fu J, Wang P, Zhang X, et al. Myeloma cells inhibit osteogenic differentiating of mesenchymal stem cells and kill osteoblasts via TRIAL induced apoptosis. Arch Med Sci 2010; 6: 496-504.

17. McLeod TL, Bechberger JF, Naus CC. Determination of a potential role of the CCN family of growth regulators in connexin 43 transfected C6 glioma cells. Cell Commun Adhes 2001; 8: 441-5.

18. Gellhaus A, Wotzlaw C, Otto T, Fandrey J, Winterhager E. More insights into the CCN3/connexin43 interaction complex and its role for signaling. J Cell Biochem 2010; 110: 129-40.

19. Ilvesaro J, Vaananen K, Tuukkanen J. Bone-resorbing osteoclasts contain gap junction connexin-43. J Bone Miner Res 2000; 15: 919-26. 
20. Ciovacco Wendy A, Goldberg CG, Taylor AF, et al. The role of gap junctions in megakaryocyte-mediated osteoblast proliferation and differentiation. Bone 2009; 44: 80-6.

21. Kanczuga-Koda L, Sulkowski S, Lenczewski A, Koda $M$, Wincewicz A, Baltaziak M. Increased expression of connexins 26 and 43 in lymph node metastases of breast cancer. J Clin Pathol 2006; 59: 429-33.

22. Chen Q, Boire A, Jin X, et al. Carcinoma-astrocyte gap junctions promote brain metastasis by cGAMP transfer. Nature 2016; 533: 493-8.

23. Grek CL, Rhett JM, Bruce JS, Ghatnekar GS, Yeh ES. Connexin 43, breast cancer tumor suppressor: missed connections? Cancer Lett 2016; 374: 117-26.

24. Sinyuk M, Alvarado AG, Nesmiyanov P, et al. Cx25 contributes to leukemia cell communication and chemosensitivity. Oncotarget 2015; 6: 31508-21.

25. Thuringer D, Berthenet K, Cronier L, Solary E, Garrido C. Primary tumor- and metastasis-derived colon cance cells differently modulate connexin expression and function in human capillary endothelial cells. Oncotarget 2015; 6: 28800-15.

26. Naus CC, Laird DW. Implications and challenges of connexin connections to cancer. Nat Rev Cancer 2010; 10: 435-41.

27. McLachlan E, Shao Q, Laird DW. Connexins and gap junctions in mammary gland development and breast cancer progression. J Membr Biol 2007; 218: 107-21.

28. Mesnil M, Crespin S, Avanzo JL, Zaidan-Dagli ML. Defective gap junctional intercellular communication in the carcinogenic process. Biochim Biophys Acta 2005; 1719 : 125-45.

29. Cancelas JA, WLM Koevoet, de Koning AE, Mayen AEM, Rombouts EJC, Ploemacher RE. Connexin-43 gap junctions are involved in multiconnexin-expressing stromal support of hemopoietic progenitors and stem cells. Blood 2000; 96: 498-505.

30. Zhang X, Sun Y, Wang Z, Huang Z, Li B, Fu J. Up-regulation of connexin- 43 in bone marrow mesenchymal stem cells plays a crucial role in adhesion and migration of multiple myeloma cells. Leuk Lymph 2015; 56: 211-8.

31. Fu JX. Cx43 expressed on bone marrow stromal cells plays an essential role in multiple myeloma cells survival and drug resistance. Arch Med Sci 2017; 13: 236-45.

32. Andrews SW, Kabrah S, May JE, Donaldson C, Morse HR. Multiple myeloma: the bone marrow microenvironment and its relation to treatment. Br J Biomed Sci 2013; 70: 110-20.

33. Attar-Schneider O, Zismanov V, Dabbah M, TartakoverMatalon S, Drucker L, Lishner M. Multiple myeloma and bone marrow mesenchymal stem cells' crosstalk: effect on translation initiation. Mol Carcinog 2016; 55: 1343-54.

34. Reagan MR, Liaw L, Rosen CJ, Ghobrial IM. Dynamic interplay between bone and multiple myeloma: emerging roles of the osteoblast. Bone 2015; 75: 161-9.

35. Cronier L, Crespin S, Strale PO, Defamie N, Mesnil M. Gap junctions and cancer: new functions for an old story. Antioxid Redox Signal 2009; 11: 323-38.

36. Plotkin LI, Lezcano V, Thostenson J, Weinstein RS, Manolagas SC, Bellido T. Connexin 43 is required for the anti-apoptotic effect of bisphosphonates on osteocytes and osteoblasts in vivo. J Bone Miner Res 2008; 23: 1712-21.

37. Plotkin LI, Bellido T. Beyond gap junctions: connexin 43 and bone cell signaling. Bone 2013; 52: 157-66.

38. Moreno AP, Lau AF. Gap junction channel gating modulated through protein phosphorylation. Prog Biophys Mol Biol 2007; 94: 107-19.
39. Behrens J, Kameritsch P, Wallner S, Pohl U, Pogoda K. The carboxyl tail of Cx43 augments p38 mediated cell migration in a gap junction-independent manner. Eur J Cell Biol 2010; 89: 828-38.

40. Belting M, Wittrup A. Nanotubes, exosomes, and nu cleic acid-binding peptides provide novel mechanisms of intercellular communication in eukaryotic cells: implications in health and disease. J Cell Biol 2008, 183: 1187-91.

41. Al-Nedawi K, Meehan B, Micallef J, et al. Intercellular transfer of the oncogenic receptor EGFRvIll by microvesicles derived from tumour cells. Nat Cell Biol 2008; 10: 619-24. 